\title{
Delayed Onset of Intraventricular Hemorrhage Following Removal of Acute Subdural Hematoma \\ - Case Report-
}

\author{
Noriaki KoJIMA, Norihiko TAMAKI, Satoshi MATSUMOTO \\ and Masayasu FuJiwARA* \\ Department of Neurosurgery, Kobe University School of Medicine, Kobe; \\ ${ }^{*}$ Department of Surgery, Fujiwara Hospital, Fukuchiyama, Kyoto
}

\begin{abstract}
A 68-year-old male presented with neurological deterioration after a lucid interval following head trauma. Computed tomographic (CT) scans on admission demonstrated a subdural hematoma in the right frontotemporal region accompanied by subarachnoid hemorrhage in the right Sylvian and interhemispheric fissures. The subdural hematoma was removed via a right frontotemporoparietal craniectomy. However, immediate postoperative CT scans revealed hemorrhage in the third and both lateral ventricles, apparently separate from the primary hemorrhages. Decompressive rupture of damaged subependymal veins is suggested as the cause of the delayed traumatic intraventricular hemorrhage.
\end{abstract}

Key words: delayed onset, head injury, intraventricular hemorrhage, subdural hematoma, subependymal vessels

\section{Introduction}

As computed tomography (CT) is a noninvasive, reliable method for detecting intracranial lesions, especially those secondary to head trauma, reports of traumatic intraventricular hemorrhage (IVH) have recently increased in number. ${ }^{2-12)}$ Traumatic IVH is usually associated with other CT lesions, such as intracerebral contusion or hemorrhage. ${ }^{2,5,7-121}$ Primary IVH is quite rare. ${ }^{2,3,6,7,12)}$ We experienced an unusual case of IVH occurring after removal of an acute subdural hematoma, which gave some interesting clues about the pathogenetic mechanism of the delayed onset of traumatic IVH.

\section{Case Report}

A 68-year-old male was hit on the right parieto-occipital region in a motorcycle accident and rapidly lost consciousness. However, he recovered about 15 minutes later and became well oriented, when ex- amination by the attending surgeon revealed no neurological deficit except for mild memory loss and a blood pressure of $138 / 82 \mathrm{mmHg}$. He went home but returned 2 hours later because of recurrent vomiting and confusion. For a neurosurgical consultation, he was referred to our hospital.

Plain skull x-ray films disclosed a right parieto-occipital fracture. CT scans demonstrated a subdural hematoma and brain contusion in the right frontotemporal area associated with subarachnoid hemorrhage in the right Sylvian and interhemispheric fissures. There was no hemorrhage in the prepontine and ambient cisterns or the ventricular system. The third and both lateral ventricles were compressed and shifted to the left (Fig. 1).

The subdural hematoma was removed by a right frontotemporoparietal craniectomy, and adequate decompression achieved. The intraoperative blood pressure was continuously monitored and remained under $150 / 100 \mathrm{mmHg}$. Postoperatively, he was comatose, and the eyes were fixed laterally with

Received November 16, 1988; Accepted September 27, 1990

Author's present address: N. Kojima, M.D., Department of Neurosurgery, Fujiwara Hospital, Fukuchiyama, Kyoto, Japan. 


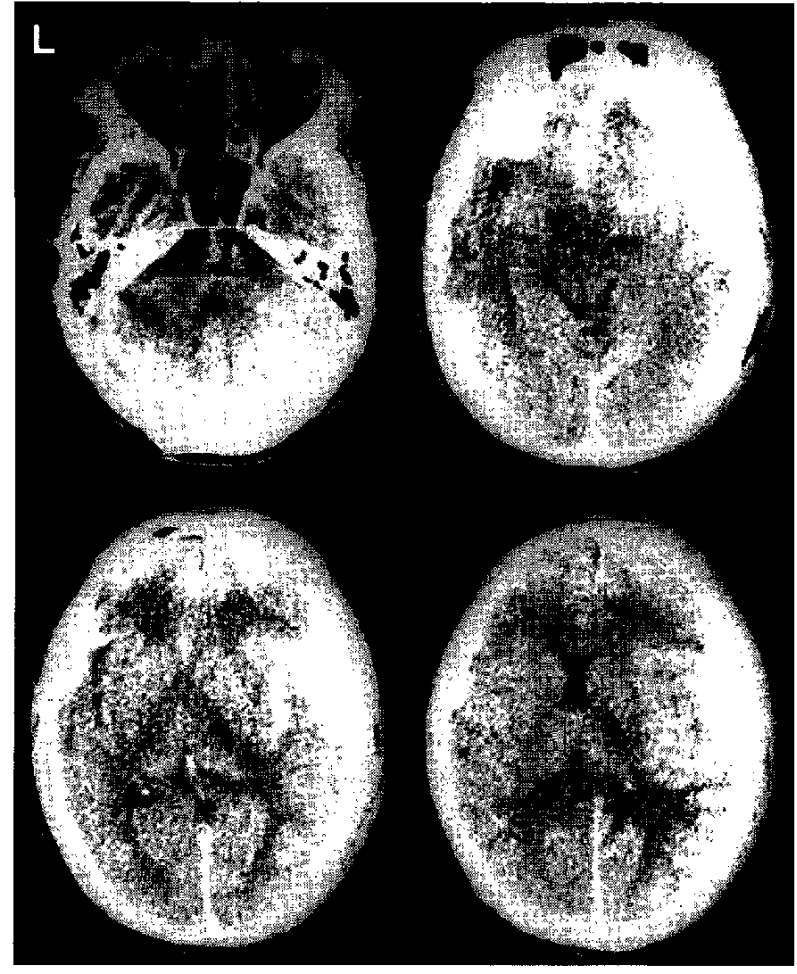

Fig. 1 CT scans on admission, showing a subdural hematoma and brain contusion in the right frontotemporal area associated with subarachnoid hemorrhage in the right Sylvian and interhemispheric fissures. The third and lateral ventricles are compressed. No intraventricular hemorrhage is present.

complete absence of the light and oculocephalic reflexes. A secondary brainstem injury was strongly suspected. Immediate $\mathrm{CT}$ scans revealed a new dense hemorrhage originating in the third ventricle and spreading into both lateral ventricles and the Sylvian aqueduct but not extending into the fourth ventricle or basal cisterns. The ventricles were dilated (Fig. 2). No vascular lesions, such as cerebral aneurysm, arteriovenous malformation, or cryptic angioma, were visualized by cerebral angiography. CT scans taken 5 hours after the operation demonstrated progressive ventricular dilatation (Fig. 3). In spite of bilateral ventricular drainage, he died 7 days after the traffic accident.

\section{Discussion}

The frequency of IVH following head trauma has been quantified since the introduction of CT (Table 1). Most cases of traumatic IVH are associated with other CT abnormalities, such as diffuse brain injury,
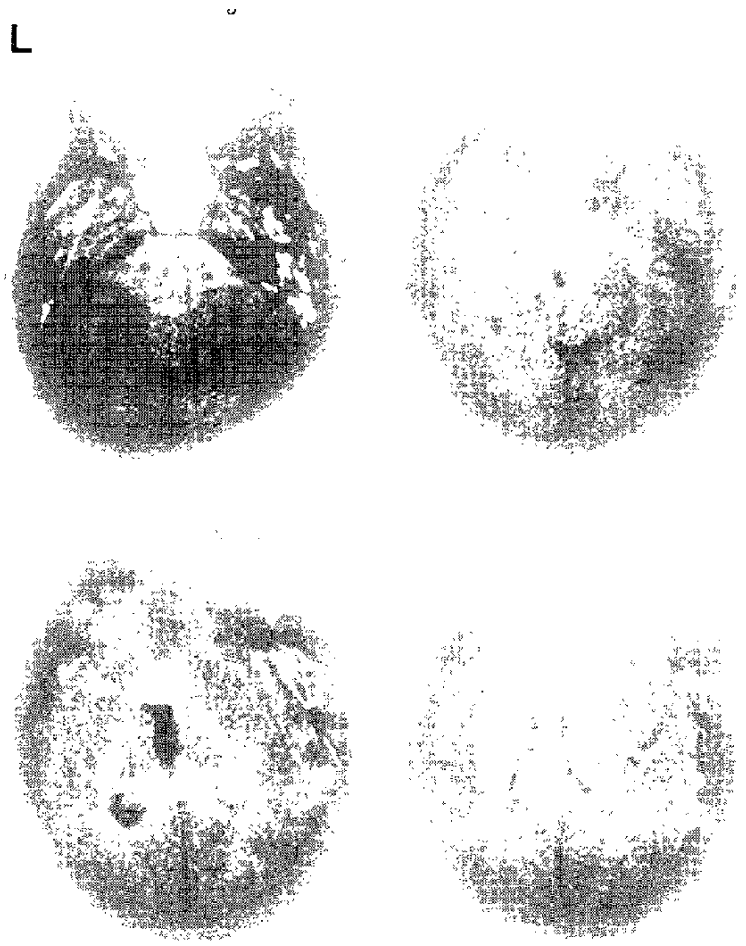

Fig. 2 CT scans immediately after the operation, showing a new dense clot in the third ventricle spreading into both lateral ventricles and the Sylvian aqueduct. The ventricles are dilated, and the brain shift is improved.

brain contusion, or subdural hematoma. However, the real mechanism of traumatic IVH has not yet been clarified. Erosion of the ventricular wall by adjacent intracerebral hematoma is the best documented cause. ${ }^{2,57.9 .12)}$ The rupture of an unsuspected periventricular vascular malformation following head trauma has also been suggested ${ }^{3,7)}$; but this is controversial, as the incidence of traumatic IVH is significantly higher than that of such arteriovenous malformations.

Our patient demonstrated neither adjacent intracerebral hematoma nor vascular lesions. The initial CT scans demonstrated a right subdural hematoma and brain contusion associated with subarachnoid hemorrhage in the interhemispheric and Sylvian fissures but no ventricular hemorrhage. The right parieto-occipital fracture and such diffuse brain damage suggest that a strong force had acted on the periventricular area or ventricular walls as well as on the right parieto-occipital region at the time of impact. ${ }^{2}$

Zimmerman et al. ${ }^{11 \prime}$ described the typical CT findings of shearing strain injuries of the cerebral 


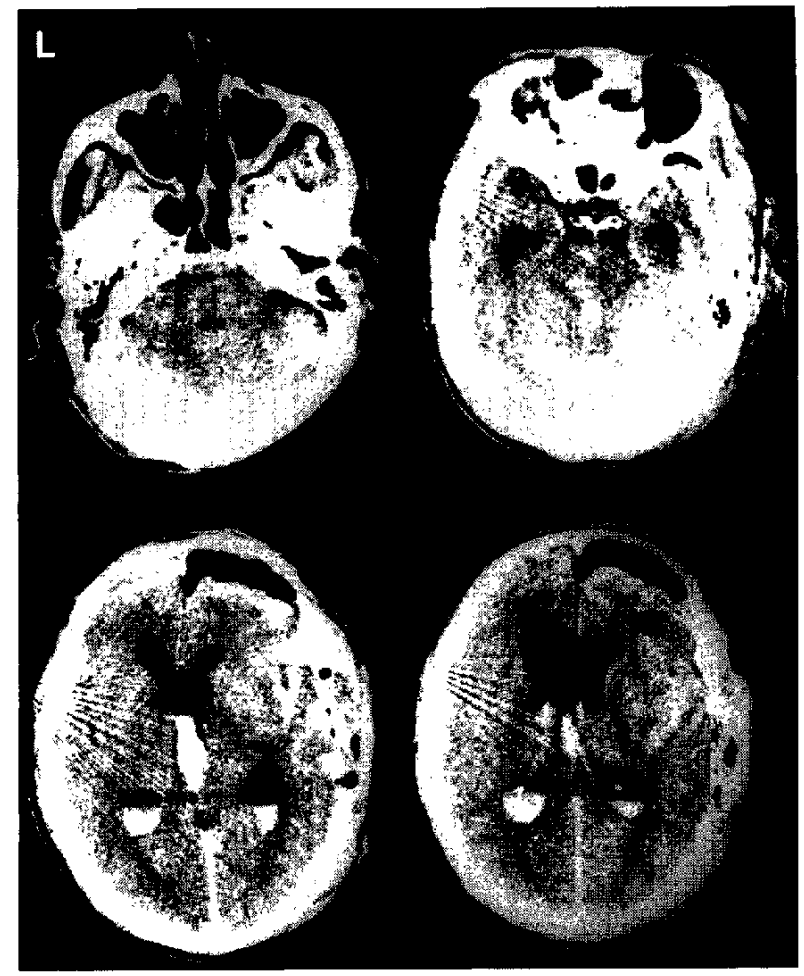

Fig. 3 CT scans 5 hours after the operation, showing a clot still occupying the whole third ventricle and the Sylvian aqueduct accompanied by progressive ventricular dilatation.

white matter as eccentric hemorrhage in the corpus callosum, diffuse brain swelling, subarachnoid hemorrhage, absence of specific focal mass lesions, and less frequent hemorrhage around the third ventricle. Tsai and Huprich ${ }^{y /}$ observed irregular contrast enhancement of the ventricular margins in one-third of traumatic IVH cases, indicating that the damaged subependymal vessels had allowed contrast material to leak into the subependymal areas.

In the present case, a shearing strain injury might have caused damage to the subependymal structures containing subependymal veins. The right subdural hematoma and consequent brain edema might have caused increased intracranial pressure (ICP) and stasis in the affected subependymal veins, thus preventing IVH. The removal of the subdural hematoma decompressed the periventricular area, resulting in rapid ventricular dilatation and rupture of the subependymal veins.

Zuccarello et al. ${ }^{12)}$ suggested such a mechanism to be the origin of traumatic IVH. Borovich et al. ${ }^{1)}$ also postulated that in head-injured patients who developed extradural hematoma within 24 hours after previous subdural or extradural hematoma removal the decompressive surgery had caused disturbances to the brain equilibrium and hemostatic effect of ICP. Intracranial hypotension and rapid recovery from peripheral vascular collapse might likewise contribute to the delayed IVH. Subependymal damage might therefore be a pathogenetic mechanism of traumatic IVH.

\section{References}

1) Borovich B, Braun J, Guilburd JN, Zaaroor M, Michich M, Levy L, Lemberger A, Grushkeiewicz I, Feinsod M, Schachter I: Delayed onset of traumatic extradural hematoma. $J$ Neurosurg 63: 30-34, 1985

2) Cordobés F, De la Fuente $M$, Lobata RD, Roger R, Pérez C, Millán JM, Bárcena A, Lamas E: Intraventricular hemorrhage in severe head injury. $J$ Neurosurg 58: 217-222, 1983

3) Danziger A, Price $\mathrm{H}$ : The evaluation of head trauma by computed tomography. J Trauma 19: 1-5, 1979

4) Dublin AB, French BN, Rennick JM: Computed tomography in head trauma. Radiology 122: 365369,1977

5) French BN, Dublin AB: The value of computerized tomography in the management of 1,000 consecutive head injuries. Surg Neurol 7: 171-183, 1977

6) Kim CH, Tanaka R, Kawamura K, Ito J: Traumatic primary intraventricular hemorrhage. Surg Neurol 16: 415-417, 1981

7) Merino-de Villasante J, Taveras JM: Computerized tomography in acute head trauma. AJR 126: 765778,1976

Table 1 Incidence of traumatic IVH and associated intracranial lesions

\begin{tabular}{|c|c|c|c|}
\hline Author (Year) & Total head trauma & IVH & Associated lesions \\
\hline Merino-de Villasante and Taveras $(1976)^{7)}$ & 100 & $3(3 \%)$ & 2 \\
\hline French and Dublin $(1977)^{5)}$ & 316 & $9(2.8 \%)$ & 9 \\
\hline Tsai and Huprich $(1978)^{91}$ & 210 & $17(8.1 \%)$ & 11 \\
\hline Oliff et al. $(1978)^{8)}$ & 200 & $6(3 \%)$ & 3 \\
\hline Danziger and Price $(1979)^{3)}$ & 133 & $2(1.5 \%)$ & 0 \\
\hline Zuccarello et al. $(1981)^{12)}$ & 350 & $10(2.9 \%)$ & 8 \\
\hline Cordobés et al. $(1983)^{2)}$ & - & $30(2.1 \%)$ & 28 \\
\hline
\end{tabular}


8) Oliff $M$, Fried AM, Young AB: Intraventricular hemorrhage in blunt head trauma. $J$ Comput Assist Tomogr 2: 625-629, 1978

9) Tsai FY, Huprich JE: Further experience with contrast enhanced CT in head trauma. Neuroradiology 16: 314-317, 1978

10) Weisberg LA: CT and acute head trauma. Comput Tomogr 3: 15-28, 1979

11) Zimmerman RA, Bilaniuk LT, Gennarelli T: Computed tomography of shearing injuries of cerebral white matter. Radiology 127: 393-396, 1978
12) Zuccarello $M$, Iavicoli $R$, Pardatscher $K$, Cervellini $\mathrm{P}$, Fiore D, Minǵrino S, Gerosa M: Posttraumatic intraventricular hemorrhages. Acta Neurochir (Wien) 55: $283-293,1981$

Address reprint requests to: N. Kojima, M.D., Department of Neurosurgery, Fujiwara Hospital, 1-38 Suehiro-cho, Fukuchiyama, Kyoto 620, Japan. 